\title{
SPECTRAL RADIUS ALGEBRAS OF WCE OPERATORS
}

\author{
Y. ESTAREMI AND M. R. JABBARZADEH
}

Abstract. In this paper, we investigate the spectral radius algebras related to the weighted conditional expectation operators on the Hilbert spaces $L^{2}(\mathscr{F})$. We give a large classes of operators on $L^{2}(\mathscr{F})$ that have the same spectral radius algebra. As a consequence we get that the spectral radius algebras of a weighted conditional expectation operator and its Aluthge transformation are equal. Also, we obtain an ideal of the spectral radius algebra related to the rank one operators on the Hilbert space $\mathscr{H}$. Finally we get that the operator $T$ majorizes all closed range elements of the spectral radius algebra of $T$, when $T$ is a weighted conditional expectation operator on $L^{2}(\mathscr{F})$ or a rank one operator on the arbitrary Hilbert space $\mathscr{H}$.

Mathematics subject classification (2010): 47A65, 47L30.

Keywords and phrases: Conditional expectation operator, spectral algebras, invariant subspace, rank one operator.

\section{REFERENCES}

[1] B. A. BARnes, Majorization, Range inclusion and factorization for bounded linear operators, Proc. Amer. Math. Soc. 133 (2004), 155-162.

[2] A. Biswas, A. LAmbert And S. Petrovic, On spectral radius algebras and normal operators, In. Univ. Math. J. 4 (2007), 1661-1674.

[3] A. Biswas, A. Lambert, S. Petrovic And B. Weinstock, On spectral radius algebras, Oper. Matrices. 2 (2008), 167-176.

[4] R. G. Douglas, Contractive projections on an $L_{1}$ space, Pacific J. Math. 15 (1965), 443-462.

[5] P. G. Dodds, C. B. Huijsmans And B. DE PAGter, Characterizations of conditional expectationtype operators, Pacific J. Math. 141 (1990), 55-77.

[6] Y. Estaremi, Some classes of weighted conditional type operators and their spectra, Positivity 19 (2015), 83-93.

[7] Y. Estaremi, On properties of Multiplication conditional type operators between $L^{p}$-space, Filomat, preprint.

[8] Y. Estaremi And M. R. JABBARZADEH, Weighted lambert type operators on $L^{p}$-spaces, Oper. Matrices 1 (2013), 101-116.

[9] J. J. GRoBler AND B. DE PAGTER, Operators representable as multiplication-conditional expectation operators, J. Operator Theory 48 (2002), 15-40.

[10] S. Jung, E. Ko And J. E. LeE, Remarks on complex symetric operators, Mediterr. J. Math. (2015), $1-10$.

[11] A. LAmbert, $L^{p}$ multipliers and nested sigma-algebras, Oper. Theory Adv. Appl. 104 (1998), 147153.

[12] A. Lamberta And S. Petrovic, Beyond hyperinvariance for compact operators, J. Funct. Anal. 219 (2005) 93-108.

[13] Shu-TEH CHEN, Moy, Characterizations of conditional expectation as a transformation on function spaces, Pacific J. Math. 4 (1954), 47-63.

[14] S. Petrovic, Spectral Radius Algebras and C Contractions, Proceedings of the American Mathematical Society 12 (2008), 4283-4288.

[15] S. Petrovic, Spectral radius algebras, Deddens algebras, and weighted shifts, Bull. London Math. Soc. 43 (2011) 513-522. 
[16] S. Petrovic, On the structure of the spectral radius algebras, J. Operator Theory. 60 (2008), $137-$ 148.

[17] S. Petrovic, Spectral radius algebras and $C_{0}$ contractions, Proc, Amer, Math. Soc. 12 (2008), 4283-4288.

[18] S. Petrovic, Spectral radius algebras and $C_{0}$ contractions II, J. Aust. Math. Soc. 89 (2010), 91-104.

[19] S. Petrovic, Spectral radius algebras and shift, Proc, Amer, Math. Soc. 5 (2010), 1639-1644.

[20] M. M. RAO, Conditional measure and applications, Marcel Dekker, New York, 1993. 